\title{
ECG SIGNAL ANALYSIS
}

\begin{tabular}{|c|c|c|}
\hline $\begin{array}{l}\text { Varsha Vatge } \\
\text { RNSIT } \\
\text { Bangalore-560098, India } \\
\text { varshavatge@gmail.com }\end{array}$ & $\begin{array}{l}\text { Ghousia Begum S } \\
\text { RNSIT } \\
\text { Bangalore-560098, India } \\
\text { ghousiabs@ gmail.com }\end{array}$ & $\begin{array}{l}\text { Vipula Singh } \\
\text { RNSIT } \\
\text { Bangalore-560098, India } \\
\text { vipulasingh@yahoo.com }\end{array}$ \\
\hline
\end{tabular}

\begin{abstract}
Accurate analysis of ECG signal is of utmost importance as the amplitudes and intervals value of ECG provide information about proper functioning of heart of every human. Therefore there have been numerous researches going on for analysis of ECG signal. In this paper we discuss about various methods that are used to extract features of ECG signal and further classify them into various disorders based on the features extracted. We also discuss about various preprocessing methods to remove base line wander and other contaminants from the ECG signal. Most researchers take input signal from MIT-BIH Data base. Performance of preprocessing is measured using Signal to Noise Ratio (SNR) and performance of feature extraction methods and classification is measured using Sensitivity and Positive Predictivity.
\end{abstract}

keywords-ECG signal, MIT-BIH Data base, feature extraction, abnormalities.

\section{INTRODUCTION}

Electrocardiograph (ECG) is a representation of electrical activity of heart. ECG signals are quasi-periodic and nonstationary by nature. It is a very important biological signal which is widely used by doctors to diagnose various heart illnesses. A single ECG cycle can be decomposed into different components, namely a $\mathrm{P}$ wave, QRS complex, a T wave and a conditional $\mathrm{U}$. Information originated in intervals and amplitudes of ECG provides valuable information of heart functional conditions.

Cardiovascular diseases (CVD) are the number one cause of death globally; more people die annually from CVDs than from any other cause. In 2012, around 17.5 million people died from CVDs, representing $31 \%$ of all global deaths. ECG signal may differ from person to person and at the same time be similar for different types of heartbeats. It is impractical and time-consuming to measure beat-by-beat manually for a long-time signal and detect the abnormalities present in them. It is difficult for a doctor to accurately find the parameters of long term ECG manually. Thus there is a need for automatic analysis of ECG signal.

Early detection of heart diseases can prolong life and enhance the quality of living through appropriate treatment. Therefore, there are numerous research and work analyzing the ECG signals. This paper provides an over view on various techniques and transformations used for preprocessing, feature extraction and abnormality detection of ECG signal.

The remainder of this paper is structured as follows. Section 2 discusses the characteristics of ECG. Section 3 discusses related work that was earlier proposed in literature for ECG preprocessing, feature extraction and abnormality detection. Section 4 describes about the results that we obtained after literature review followed by conclusion in Section 5.

\section{ECG CHARACTERISTICS}

The ECG device detects and amplifies the tiny electrical changes on the skin that are caused when the muscle depolarizes during each heartbeat. One cardiac cycle consists of P-QRS-T sequence as shown in fig. 1. It has a conditional $\mathrm{U}$ wave, which is normally invisible in 50 to $75 \%$ of ECGs because it is hidden by the $\mathrm{T}$ wave and upcoming new $\mathrm{P}$ wave. $P$ wave indicates atrial depolarization or contraction of the atrium, QRS complex indicates ventricular depolarization, or contraction of the ventricles and $\mathrm{T}$ wave indicates ventricular repolarization. Normally, the frequency range of an ECG signal is of $0.05-100 \mathrm{~Hz}$ and its dynamic range is of $1-10 \mathrm{mV}$.

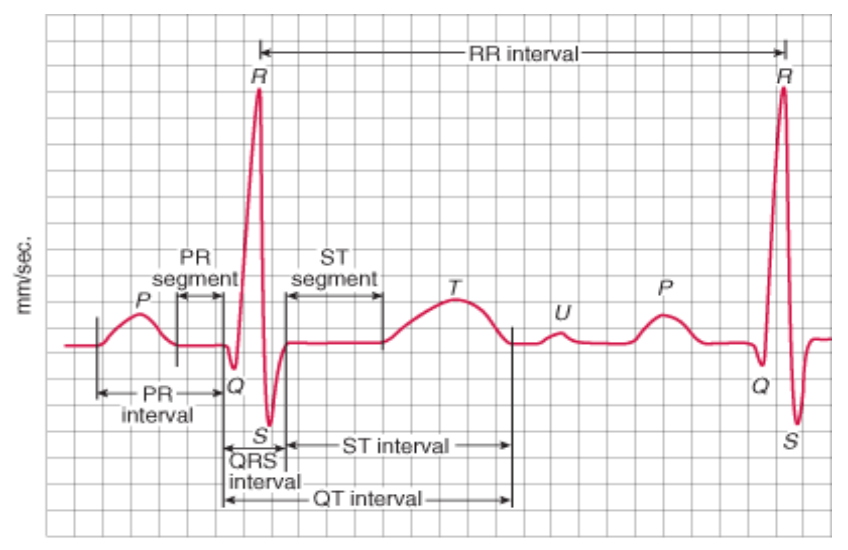

Fig. 1 ECG signal

The normal value of heart beat lies in the range 60 to 100 beats/minute. A slower rate than 60 beats per minute is called 


\section{ELK Asia Pacific Journals - Special Issue}

ELK

Asia Pacific Journals

bradycardia (Slow heart) and a higher rate than 100 beats per minute is called tachycardia (Fast heart).

Amplitude

Duration

$\begin{array}{llll}\text { P-wave } & : 0.25 \mathrm{mv} & \text { P-R interval } & : 0.12 \text { to } 0.20 \mathrm{~s} \\ \text { R-wave } & : 1.60 \mathrm{mv} & \text { Q-T interval } & : 0.35 \text { to } 0.44 \mathrm{~s} \\ \text { Q-wave } & : 25 \% \mathrm{R} \text { wave } & \text { S-T interval } & : 0.05 \text { to } 0.15 \mathrm{~s} \\ \text { T-wave } & : 0.1 \text { to } 0.5 \mathrm{mv} & \text { P-wave interval } & : 0.11 \mathrm{~s} \\ & & \text { QRS interval } & : 0.09 \mathrm{~s}\end{array}$

ECG signal recordings present in MIT-BIH are broadly classified into 3 groups- Normal, Premature Ventricular Contraction (PVC) and Atrial Premature Complexes (APC). Normal ECG is an electrocardiogram without a recognizable abnormality. PVC can be perceived as a "skipped beat" or felt as palpitations in the chest. A single beat PVC arrhythmias do not usually pose a danger and can be asymptomatic in healthy individuals. APC, also known as atrial premature beats (APB), are a common cardiac dysrhythmia characterized by premature heartbeats originating in the atria

\section{ECG signal Analysis}

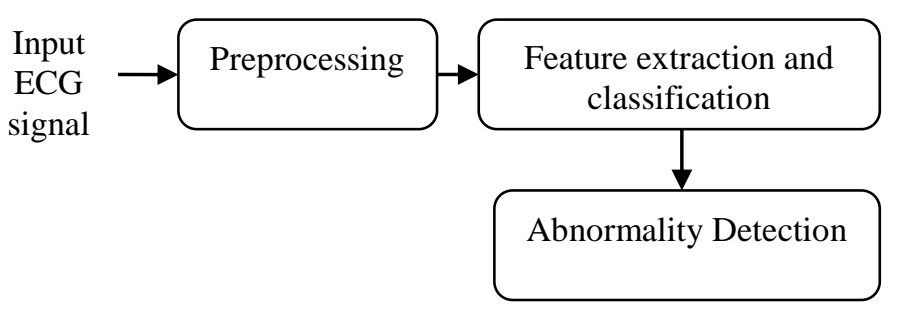

Fig. 2 System level Block Diagram

Input ECG signal is taken from MIT-BIH database [25]. The database contains 48 two-channel records which are sampled at frequency of $360 \mathrm{~Hz}$. Each recording has duration of 30 min and includes two leads - the modified limb lead II and one of the modified leads V1, V2, V4 or V5. ECG data (12 ECG leads) can also taken from Physikalisch Technishe Bundesanstalt (PTB) diagnostic ECG database (PTB-DB) [24].

The ECG signal is usually contaminated with various noises such as power line interference, baseline drift, electrode contact noise, motion artifact, muscle contraction, Electromyographic(EMG) interference.[25]

Power line interference: Power line interference consists of $60 / 50 \mathrm{~Hz}$ pickup and harmonics and the amplitude is $50 \%$ of peak-to-peak ECG amplitude.
ISBN: 978-81-930411-5-4

Baseline Drift: Caused by respiration or patient movement which creates problems in the detection of peaks. Due to wander $\mathrm{T}$ peak would be higher than $\mathrm{R}$ peak which might be detected as R peak instead. Amplitude variation is $15 \%$ of peak to peak ECG amplitude.

Electrode contact noise: The connection between patient and measuring system is interrupted for a short duration due to improper contact of the electrodes which creates electrode contact noise which is of duration $1 \mathrm{sec}$ and amplitude of which is maximum recorded output of ECG signal with frequency of $60 \mathrm{~Hz}$.

Motion artifact: Transient base line changes caused by changes in the electrode-skin impedance with electrode motion due to movement of the patient while the ECG is being recorded. Duration of this noise is $100-500 \mathrm{~ms}$ with amplitude of $500 \%$ peak to peak ECG amplitude.

Muscle contractions: Also called as EMG (electromyography) noise which is induced by the patient's movement and is responsible for artifactual milli-volt level potentials to be generated [25].

ECG signal preprocessing is to remove these contaminants from it and obtain valid signal component. As the ECG recordings are subjected to different kind of noises which lie in different frequency ranges, a single filter cannot be used for noise removal.

Preprocessed signal is used to further extract features from ECG signal. The ECG feature extraction system provides fundamental features (P-QRS-T) which is used for further classification. ECG features can be extracted in time domain or frequency domain.

The section is divided into three main parts.

1. Preprocessing.

2. Feature extraction and classification.

3. Abnormality detection.

Each part's related work is discussed which was being proposed earlier in Literature.

\section{A. Preprocessing}

Preprocessing is done to remove noises that bring obstacle to the accurate diagnosis of ECG signal. Proper classification could not be done if there are contaminants in the input signal.

A huge number of efficient techniques have been reported in the literature addressing the issue of noise elimination from digitized ECG signal such as Wavelet Based Filter [1], Band 


\section{ELK Asia Pacific Journals - Special Issue}

ELK

Asia Pacific Journals

ISBN: 978-81-930411-5-4

Pass Filter [2], Weighted Averaging filter [6], Artificial Neural Network [4], Adaptive filter [5], Principal Component analysis [27], Independent Component Analysis [26], Empirical Mode Decomposition [8] and Kalman Filter [3].

Arman Sargolzaei et al. [1] used multi-resolution based on wavelet transform for suppression of base line wander. The technique extracts the model of baseline wandering and then uses the model to reconstruct the corrected ECG signal. The proposed technique removes baseline wandering, while preserving the clinical information and the morphology of the ECG record. Moreover the proposed technique is also capable of improving signal-to-noise ratio while eliminating the baseline wandering.

Deng et al. [2] proposed the ECG Mask-Filtering Method for rapid removal of ECG interference in diaphragmatic electromyographic (EMGdi) signal which is also referred as muscle contraction noise in ECG. It consists of a QRS trigger, wide hanning window, pass filters \& signal synthetizer. It consists of a Butterworth three-order $60 \mathrm{~Hz}$ highpass filter which strongly attenuates the $\mathrm{P}$ and $\mathrm{T}$ waves of the ECG so that their effects on the detection of the EMG can be neglected. Thus the QRS-complexes were removed over the mask durations.

MA Mneimneh et al [3] used the kalman filter (KF) approach to identify \& remove baseline wandering in ECG signals without adding any distortion to the signal. First a state space model is derived through equations \& the solution for that model is determined using an iterative approach. But this KF approach for the removal of baseline wandering failed for the condition under high frequency changes due to adaptability \& convergence factor of KF.

Mateo \& Sachez. [4] worked on elimination of baseline wandering by using neural networks. The proposed system is based on a grown Artificial Neural Network (ANN) which allows optimization of both the hidden layer number of nodes and the coefficient matrixes.

Alireza K. Ziarani \& Adalbert Konrad. [5] proposed a new adaptive electromagnetic interference (EMI) filter for power line noise removal from ECG. Their EMI filters core algorithm can be employed to extract the power line signal mixed with the ECG signal. A single unit of their algorithm can effectively follow time variations in amplitude, phase and frequency of the interfering signal. Once it is extracted, it can be subtracted from the input signal to yield a clean ECG signal.

Steven J. Simske \& Daniel R. Blakley [6] proposed a new method for noise removal. First ECG is converted to vectorcardiogram (VCG) signal, a simple average filter is used to remove the unwanted noises and then VCG is converted back to ECG. It was very effective in removing
$60 \mathrm{~Hz}$ and random noise in the ECG and data received after filtering is of high clinical value. Due to the low frequency nature of the VCG, an analysis of variability (both of magnitude \& direction) may be performed in either the time domain or the frequency domain.

Patil \& Chavan [7] adopted Daubechies wavelet (Orthogonal wavelet) for removal of power line interference and baseline wander from ECG signal. They used a Daubechies wavelet with a decomposition tree of level 5 as it can provide a well orthogonality to high frequency noise with a given number of vanishing moments. Excellent performance was observed by them in mitigating power line interference on the ECG signals. The advantages being good interpretation of both local scale and time aspects of a given signal making the wavelet transform an effective method for time-frequency analysis of non-stationary signals.

Hongqiang $\mathrm{Li}$ et al. [8] presented a new ECG signal processing approach based on empirical mode decomposition (EMD) and an improved approximate envelope method. They used Butterworth low pass filter used to eliminate high frequency noises before applying EMD. They could also correct baseline wandering and eliminate low frequency noises by eliminating last two-order intrinsic mode functions.

\section{B. Feature extraction}

There have been several algorithms and methods proposed for accurate feature extraction since many years. Some methods used are Wavelet transform method- discrete wavelet [16], Daubechies , quadratic spine wavelet [10], Mean- shift algorithm [11], Discrete cosine transform [12], Hilbert Transform [13], Time Domain Based Classifier [14], Time series alignment [15], Empirical mode decomposition [9].

Md. Abdullah Arafat and Md. Kamrul [9] have used Empirical Mode decomposition method (EMD) for detecting ECG characteristic wave. The EMD is a powerful tool for analysing nonlinear and non-stationary data. The aim of EMD is to decompose the signal into a sum of intrinsic mode functions (IMFs). QRS complex is detected from lower order IMFs and $\mathrm{P}$ waves and $\mathrm{T}$ waves are detected from high order IMFs.

Mohammad Niknazar et al. [10] have used waveletbased method for detection of characteristic of ECG signal. Quadratic spine wavelet is chosen as mother wavelet in the proposed algorithm. The proposed method is applied directly to the original signal without any filtering or pre-processing. It is robust to the baseline shifts and to dominant noise. The proposed method is not able to find onset-offset of $\mathrm{P}$ and $\mathrm{T}$ waves in severe noisy cases.

Jingyu Yan et al. [11] proposed a mean shift based approach for ECG feature detection with a robust 


\section{ELK Asia Pacific Journals - Special Issue}

ELK

Asia Pacific Journals

ISBN: 978-81-930411-5-4

performance to kinds of noisy signals. They generated clean ECG signal by using ECG synthesizer (ECGSyn). Noises of white, pink and brown colors are further added to synthetic signal for experimenting. The time consumption of the proposed algorithm is comparatively high so that it can be only used as an off-line tool.

Somsanuk Pathoumvanh et al. [12] have used Discrete Cosine Transform (DCT) for feature extraction. The paper proposed uses the power spectral of DCT coefficients for analysis and the DCT transformation property is utilized to reset the equality of each flexible ECG beat length, by omitting the powerless part of the DCT coefficients.

S.Dhar et al. [13] have implemented Lagrange Five Point interpolation formula on ECG signal after de-noising. R peaks are found using Hilbert transform. The Q and S waves were located by searching minimum amplitude on either sides of the R-peak.

G.P. Shorten and M.J. Burke [14] have used dynamic time warping (DTW) for pattern recognition .The DTW process can take two signals of different frame length and duration and align them using a non-linear dynamic process. The authors have suggested a classifier that uses a time domain based combination of features including heart rate, degree of time alignment required and an amplitude difference measurement.

Bachir Boucheham [15] has proposed method based on a pattern recognition approach through usage of a time series comparison and alignment technique. Shape exchange algorithm (SEA) is used to detect the morphology changes in the ECG. The author has performed segment-wise comparisons of the ECG where one segment stands for the reference (normal) behavior and the other segment for the unknown behavior segment. Based on co-relation factor, that reflects the degree of similarity between the reference and the segment under analysis, the whole segment is classified as normal or abnormal.

Abdulrhman Elbuni et al. [16] have used discrete wavelet transform method for accurate estimation of ECG parameters. The wavelet transformation is based on a set of analyzing wavelets allowing the decomposition of ECG signal in a set of coefficients. Within each level (or scale) the signal is decomposed in two parts: the detail, related with the high frequencies, and the approximation, containing the information of the low frequencies. This last part is processed in a similar way in the next level, and so on. In this way it is possible to decompose the signal information (according to the frequency bands) in the detail levels, allowing a multi resolution (or multi scale) analysis.

Antonio Espiritu-Santo-Rincon [17] presented an algorithm for detecting duration and amplitude of the ECG individual components. First the $\mathrm{R}$ wave is precisely detected using wavelets, and then the other ECG features are extracted using a waveform segmentation approach. First the $\mathrm{R}$ wave is precisely detected using wavelets. The other ECG features are extracted using a waveform segmentation approach.

\section{Abnormalities}

There have been various methods proposed for abnormality detection. Few abnormalities that are recognized includes -Normal Sinus Rhythm (NSR), Atrial Premature Contractions (APC), Premature Ventricular Contractions (PVC), Ventricular Fibrillation (VF), Ventricular Tachycardia (VT) and Supra Ventricular Tachycardia (SVT).

Classifier methods include hybrid neuron network, support vector machine (SVM), extreme learning machine (ELM), and Type-2 fuzzy clustering neuron network. Here we review few methods for identifying abnormalities.

X. Tang and L. Shu [18] proposed classifier based on QNN (Quantum Neural Network). Quantum Neural Network (QNN) is a youthful and energetic science built upon the combination of quantum computing and artificial neural network. It also combines the advantages of neural modelling and fuzzy theoretic principles. Authors could reduce the dimension of feature space and decrease the complexity in the process by the proposed method.

Rizwan R. Sheikh and Imtiaz A. Taj [19] have classified cardiac rhythms/segments of ECG based on statistical and morphological features extracted from the ECG signal. Classification was done using Euclidian Manhalanobis Distance classifiers. They used Principle Component Analysis (PCA) and Linear Discriminant Analysis (LDA) for dimensionality reduction. Using this method, authors could diagnose five cardiac conditions namely Normal (N), Ventricular Flutter (VFL), Ventricular Tachycardia (VT), Nodal (NOD) and Left Bundle Branch Block (L).

Pratiksha Sarma et al [20] have classified the beats using MLP (multi layer perceptron) Neural Network. In the classification stage, they have proposed Feed-Forward network with one input layer, one hidden layer and one output layer. In the paper, five types of arrhythmias are considered: normal beat (NORMAL), premature ventricular contraction (PVC), paced beat (PB), Right Bundle Branch Block (RBBB) and Left Bundle Branch Block (LBBB).

Ashutosh Gupta et al [21] have presented a non-linear neural network model for ECG classification. They have used two layered feed-forward back-propagation network with sigmoid output neurons in order to grade the input ECG dataset into four different signal classes: Myocardial Ischemia 
class, Atrial Fibrillation class, Ventricular Tachyarrhythmia class and healthy subjects.

Qibin Zhao and Liqing Zhang [22] have used support vector machine (SVM) with Gaussian kernel to classify different ECG heart rhythm. The SVM used for classification of the ECG beat was trained, cross validated and tested with the extracted features from discrete wavelet transform and Auto Regressive (AR) model of the ECG signals. The classifier proposed in the paper is a learning machine of the supervised type and Multi category SVM (MC-SVM).

\section{RESULTS}

This review summarizes the various Preprocessing, feature extraction and Abnormality detection papers. Input ECG signal is taken from MIT- Database or PTB- Database $[23,24]$. ECG signal can also be simulated in MATLAB.

Baseline wander is a common phenomenon in ECG recordings taken from any database. Presence of baseline wander leads to inaccurate analysis of signal. Wavelet transform based methods are more effective in removing baseline wander than conventional filtering methods. First advantage being FIR filters have time dilation and this property can be affected the proficiency in real time applications. Another advantage of the wavelet is that it creates less distortion on the signal than FIR filters [1]. Fig. 3 shows input signal before and after removal of Baseline wander.

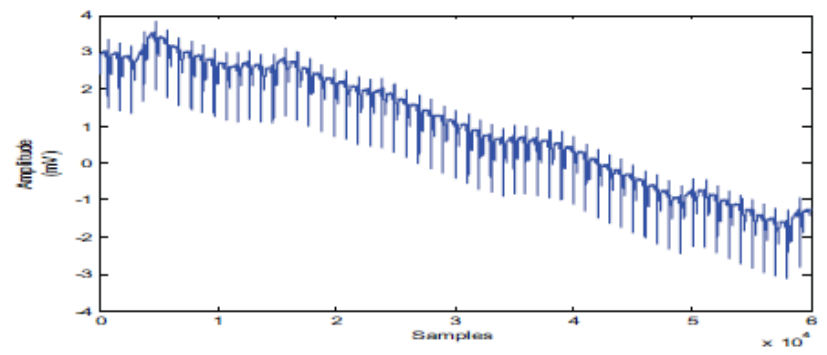

(a)

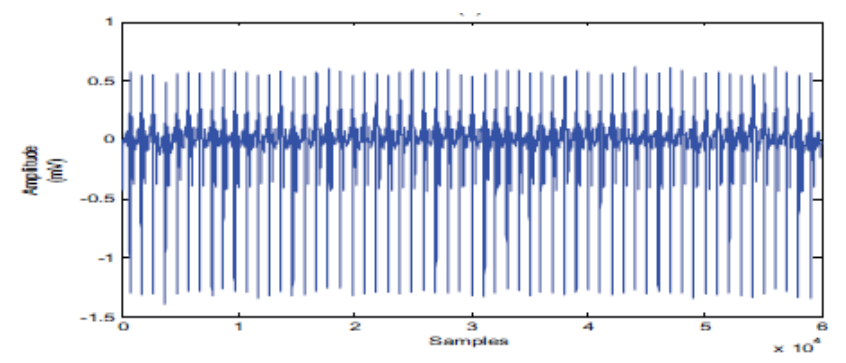

(b)

Fig. 3 (a) Distorted signal by baseline wandering (b) Signal after removal of baseline wandering [1]
ECG signal analysis could be either done in time domain or frequency domain after preprocessing. Feature extraction is also done using pattern recognition and various algorithms and transformation methods. Commonly used parameters for comparison includes Sensitivity (Se), Positive Predictivity (PP) and Detection Error Ratio (DER).

$$
\begin{aligned}
& \mathrm{Se}(\%)=\mathrm{TP} / \mathrm{TP}+\mathrm{FN} \\
& \mathrm{PP}(\%)=\mathrm{TP} / \mathrm{TP}+\mathrm{FP} \\
& \mathrm{DER}=(\mathrm{FP}+\mathrm{FN}) / \text { Total Beats }
\end{aligned}
$$

True Positive (TP) represents correctly detected beat, False Negative (FN) represents missed detection and False Positive (FP) represents extra detection.

We compared sensitivity of different methods used for feature extraction in Table I and found that best results were obtained using Quadratic spline wavelet transform and Hilbert transform method [10, 13]. Empirical mode decomposition gave $100 \%$ sensitivity for QRS complex detection [9].

TABLE I. Comparison of sensitivity for different feature extraction methods

\begin{tabular}{|c|c|c|}
\hline Author & Feature Extraction Method & $\begin{array}{c}\text { Sensitivity } \\
(\boldsymbol{\%})\end{array}$ \\
\hline $\begin{array}{c}\text { Abdulrhman } \\
\text { Elbuni[16] }\end{array}$ & Discrete Wavelet transform & 89.2 \\
\hline $\begin{array}{c}\text { Mohammad Niknazar } \\
\text { et al.[10] }\end{array}$ & $\begin{array}{c}\text { Quadratic Spline wavelet } \\
\text { transform }\end{array}$ & 99.84 \\
\hline Somsanuk et al.[12] & Discrete Cosine Transform & 97.01 \\
\hline $\begin{array}{c}\text { S.Dhar et al.[13] } \\
\text { Hilbert Transform }\end{array}$ & 99.84 \\
\hline $\begin{array}{c}\text { Md. Abdullah and Md. } \\
\text { Kamrul [9] }\end{array}$ & $\begin{array}{c}\text { Empirical Mode } \\
\text { Decomposition(QRS complex) }\end{array}$ & 100 \\
\hline
\end{tabular}

Abnormalities are detected after feature extraction of the ECG signal. ECG signal is classified into various classes based on abnormalities present in them. Fig. 4 shows ECG signal classified by five types of arrhythmias: normal beat (NORMAL), premature ventricular contraction (PVC), paced beat $(\mathrm{PB})$, Right Bundle Branch Block (RBBB) and Left Bundle Branch Block (LBBB) [20].

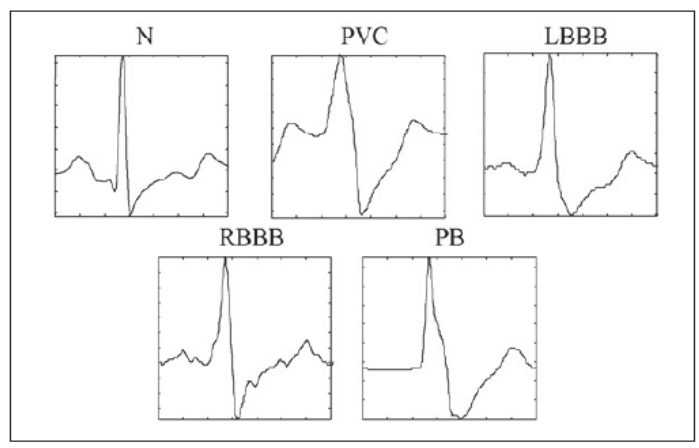




\section{ELK Asia Pacific Journals - Special Issue}

ISBN: 978-81-930411-5-4

\section{Asia Pacific Journals}

Fig. 4 Reference waveforms for the five heartbeat types under classification (N, PVC, LBBB, RBBB, PB) [20]

\section{CONCLUSION}

The examination of the ECG has been comprehensively used for diagnosing many cardiac diseases. This proposed paper provides an overview of various techniques and algorithms for preprocessing feature extraction and classification of ECG signal. Wavelet based method is widely used for removing baseline wander and different types of noise. The wavelet transform is an effective method for timefrequency analysis of non-stationary signals. It has benefits of good interpretation of both local scale and time aspects of a given signal.

We compared various methods used for feature extraction based on sensitivity. Quadratic spline wavelet transform and Hilbert transform method gave high sensitivity for feature extraction. Sensitivity and Positive predictivity should be high and Detection error rate should be zero for accurate analysis. No proposed method gave $100 \%$ sensitivity and positive predictivity for overall detection. Future scope lies in achieving these factors for accurate analysis.

\section{REFERENCES}

[1] Arman Sargolzaei et al., "A New Robust Wavelet Based Algorithm for Baseline Wandering Cancellation in ECG Signals", IEEE International Conference on Signal and Image Processing Applications, 2009

[2] Deng Y.-C "On-line cancelling technique of ECG interference in the diaphragmatic electromyographic signals", IEEE, 1997

[3] MA Mneimneh et al, "An Adaptive Kalman Filter for Removing Baseline Wandering in ECG Signals", Computers in Cardiology, 2006

[4] J. Mateo et al., "Neural Networks Based Approach to remove Baseline drift in Biomedical Signals", IFMBE Proceedings 16, pp. 90-93, 2007

[5] Alireza K. Ziarani \& Adalbert Konrad , "A Nonlinear Adaptive Method of Elimination of Power Line Interference in ECG Signals", Ieee transactions on Biomedical Engineering, vol. 49, no. 6 , june 2002

[6] Steven J. and Daniel R, "Using the vectorcardiogram to remove ecg noise", IEEE, 2012

[7] Pradnya B. Patil and Mahesh S. chavan, "A Wavelet Based Method for Denoising of Biomedical Signal" Proceedings of the International Conference on Pattern Recognition, Informatics and Medical Engineering , 2012

[8] Hongqiang $\mathrm{Li}$ et al., "Denoising And R-peak Detection Of Electrocardiogram Signal Based On Emd And Improved Approximate Envelope", Springer Science+business Media New York 2013

[9] Md. Abdullah Arafat1 et al, "Automatic Detection of ECG wave Boundaries Using Empirical Mode Decomposition”, IEEE 2009
[10] Mohammad Niknazar et al., "Detection of Characteristic Points of ECG using Quadratic Spline Wavelet Transfrom”, International Conference on Signals, Circuits and Systems, 2009

[11] Jingyu Yan et al., "Model-based Feature Extraction of Electrocardiogram Using Mean Shift", IEEE

[12] Somsanuk Pathoumvanh et al., "Arrhythmias Detection and Classification base on Single Beat ECG Analysis", Electronic and Electrical Engineering (JICTEE-2014)

[13] S. Dhar et. Al "Noise Reduction and ECG Feature Extraction Using Interpolation and Hilbert Transform", International Conference on Control, Instrumentation, Energy \& Communication, 2014

[14] G.P. Shorten et. al, "A Time Domain Based Classifier for ECG Pattern Recognition", 33rd Annual International Conference of the IEEE EMBS August 30 - September 3, 2011

[15] Bachir Boucheham, "Abnormality Detection in Electrocardiograms by Time Series Alignment ", CISME Vol.1 No.3 2011

[16] Abdulrhman Elbuni et al., "ECG Parameter Extraction Algorithm using (DWTAE) Algorithm", International Conference on Computer Technology and Development, 2009

[17] Antonio et al., "ECG Feature Extraction via Waveform Segmentation", 7th International Conference on Electrical Engineering, Computing Science and Automatic Control (CCE 2010)

[18] X. Tang and L. Shu, "Classification of Electrocardiogram Signals with RS and Quantum Neural Networks", International Journal of Multimedia and Ubiquitous Engineering Vol.9, No.2 (2014)

[19] Rizwan R. Sheikh and Imtiaz A. Taj, "Cardiac Disorder Diagnosis Based on ECG Segments Analysis and Classification", IEEE, 2009

[20] Pratiksha Sarma et al, "ECG Classification using Wavelet Sub band Energy based Features", International conference on signal processing and integrated network (SPIN), 2014

[21] Ashutosh Gupta et al, "Neural Network based Indicative ECG Classification", IEEE, 2014

[22] Qibin Zhao and Liqing Zhang, "ECG Feature Extraction and Classification Using Wavelet Transform and Support Vector Machines", IEEE, 2005

[23] http://www.physionet.org/physiobank/database/ptbdb/

[24] http://www.physionet.org

[25] Sarang L. Joshi et al, "A Survey on ECG Signal Denoising Techniques", International Conference on Communication Systems and Network Technologies, 2013

[26] Mrinal Phegade et al, , “ ICA Based ECG Signal Denoising”, IEEE, 2013

[27] Francisco Castells et al, "Principal component analysis in ECG signal processing", Hindawi publishing Corporation, EURASIP Journal on Advances in signal Processing, Vol. 2007, 2006. 